\title{
Role of Statins in Breast Cancer Management: Current Issues and Future Directions
}

\author{
* Bader A. Abdelmaksoud \\ Department of Clinical Oncology and Nuclear Medicine, Faculty of Medicine, Zagazig University, Egypt
}

Submission: March 21, 2017; Published: March 27, 2017

"Correspondence Address: Bader A. Abdelmaksoud, Department of Clinical Oncology and Nuclear Medicine, Faculty of Medicine, Zagazig University, Egypt

\begin{abstract}
3-hydroxy-3-methylglutaryl coenzyme A (HMG-CoA) reductase inhibitors, or statins, are commonly-used drugs as cholesterol lowering medications in patients with hypercholesterolemia or cardiovascular disorders. Beyond cholesterol lowering properties, statins also exert pleiotropic actions. In certain breast cancer cell lines, preclinical studies demonstrated that statins have anti- proliferative properties with apoptosis induction properties and decrease in the invasion and metastasis. Also, statins may have a role in treatment of highly aggressive types (e. g., inflammatory breast cancer and triple-negative type) known to have poor prognosis. In metastatic bone disease, there are evidences of their beneficial role in prevention of bone resorption. The major advantage of statins is that it is inexpensive, well tolerated and hence it is considered to be cost effective agents in cancer treatment. So, incorporation of statin in breast cancer treatment should be encouraged in future randomized control studies.
\end{abstract}

Keywords: Breast cancer; Statins; HMG-CoA Reductase Inhibitors

\section{Introduction}

Statins are inhibitors of 3-hydroxy-3-methylglutaryl coenzyme A (HMG-CoA)reductase, commonly used as cholesterol lowering drugs [1]. Beyond the cholesterol lowering function, statin exert pleiotropic actions as stabilization of atherosclerotic plaque, reduction of vascular inflammation, and some degrees in reduction of short term recurrent ischemia in coronary heart diseases. Also,they have immunomodulatory effects and sometimes are beneficial in autoimmune conditions. Antiproliferative effects on smooth muscles and stimulation of bone marrow, also have been recorded [2,3]. In cancer, Statins interrupt the mevalonate pathway which affect many downstream signals responsible for cancer growth and progression [4]. Both pre-clinical and clinical studies have shown that statins have anti-tumor effect in a variety of tumor cell lines, including melanoma, glioma, neuroblastoma, and leukemia cell lines [5]. Also, both pre-clinical and clinical trials support their possible benefits in breast cancer [6].

\section{Pharmacology}

HMG-CoA Reductase (HMGCA) is a rate limiting enzyme in mevalonate pathway. Statins are competitive inhibitors of this enzyme. By inhibition of HMGCR, mevalonate synthesis is inhibited and subsequently its downstream signals are arrested [6]. Pharmacodynamically, after oral intake, about $30 \%$ to $80 \%$ of the drug is absorbed in the intestine, then, it undergoes extensive first-pass hepatic uptake in which biotransformation is occurred with elimination of more than $70 \%$ of drug metabolites [7]. The peak plasma concentration was reached after 1 - 4 hours after drug intake with different half -life of the original drug, in atorvastatin and rosuvastatin for example it is $20 \mathrm{~h}, 12 \mathrm{~h}$ in simvastatin and ranged 1-4 h for other statins[8]. Regarding the solubility, the hydrophilic statins (lovastatin) are limited to the liver, but the lipophilic ones (atorvastatin) have extrahepaticpermeation, this may explain why many studies showed that the lipophilic statins are more effective in cancer treatment greater than hydrophilic ones $[9,6]$.

\section{Statins and Cellular Proliferation}

As known, Mevalonate is a precursor of various cell cycle regulating compounds (e.g., geranylpyrophosphate (GPP), farnesyl pyrophosphate (FPP) and dolichol which are linked to DNA synthesis and subsequent several tumor proteins production as Ras and Rho, which involved in regulation of cellular signal transduction of some membrane receptor proteins critical for gene transcription involved in cellular proliferation, differentiation, and apoptosis [5,10]. The preclinical studies showed that statins cause inhibition of GPP, FPP and dolichol production with subsequent arrest of tumor cell proliferation [11]. In breast cancer, statins Inhibit cellular proliferation by interfering with different ways of these pathways, this was explained in various studies. Statins were supposed to exert their 


\section{Cancer Therapy \& Oncology International Journal}

action by inhibition of RhoA-like GTPases which are appeared to be more expressed in breast tumors rather than in normal cells, also the RhoA-like proteins amount was associated with other prognostic factors as tumor grade and proliferative indices [12]. Keyomarsi et al. in their study demonstrated that statins cause FPP and GGPP depletion and disrupt protein prenylation with subsequent inhibition of proliferation in cancer cells [13]. Kanugula et al. [14] investigated in their study the effect of lipophilic statins on nuclear factor kappa B (NFкB) which isa transcription factor when activated increase proliferation and reduce apoptosis. They found that lipophilic statins deactivate $\mathrm{NF \kappa B}$ with subsequent inhibition of proliferation in some breast cancer cell lines. Also, they observed that breast cancer cells with Ras or cErbB2 expression had higher amount of activated $\mathrm{NF}-\kappa \mathrm{B}$ than those with estrogen receptor expression, and so, it may be more sensitive to statins. So, statins may have benefits in the treatment of these types of breast cancer as triple negative type [14]. The anti-proliferative effect of statins in breast cancer was confirmed in various studies by detecting the changes of proliferative indices before and after statins administration. The proliferation of breast cancer cells was assessed by changing of Ki 67 pre-andpost-statins intake as shown on study conducted by Bjarnadottir et al [15]. Similar results were obtained in other studies confirming the anti-proliferative effect of statins on breast cancer tissues [16-24].

\section{Anti-Angiogenesis Effect}

In several studies, the relation of statins and angiogenesis was investigated and variable results were obtained. In some studies, statins were supposed to be inhibitors of angiogenesis and decrease tumor vascularization and endothelial proliferations $[17,18]$. Other studies showed different results in which statins appeared to be angiogenesis stimulators through nitric oxide synthase activation with subsequent endothelial proliferation [19]. There is another opinion in which the effect of statins onthe endothelium is dose related, as shown in Weis et al. [17] study in which they demonstrated that low dose of statins $(0.5 \mathrm{mg} / \mathrm{kg} /$ day)stimulate endothelial cell proliferation, but higher dose $(2.5$ $\mathrm{mg} / \mathrm{kg} /$ day) showed significant inhibition of angiogenesis [17]. So, the anti-angiogenic effect of statins needs further researches to be clarified and this effect should be investigated in both tumors and normal tissue variants models.

\section{Statins and Apoptosis}

Various experimental cancer studies have demonstrated that statins have apoptotic properties in several tumor cell lines, and these effects were seen in both solid and hematologic malignancies [5]. In breast cancer, extensive studies were undertaken to investigate the effect of statins on apoptosis in breast cancer cell lines, Koyuturk et al. [20] showed that statins cause apoptosis in breast cancer cells by activation of Jun $\mathrm{N}$-terminal kinases (JNK) signaling pathways, they also observed that effect was independent ofER or p53 status [20]. Interestingly, the same results obtained in other studies conducted by
Brozovic et al and Brantley-Finley et al, they found in their studies the resistance to chemotherapeutic drugs as vinblastine and cisplatin results from inhibition of JNK activation, so statins may decrease this resistance to these agents. They also observed that, statins(simvastatin) caused cell-cycle arrest, apoptosis and JNK cellular signaling activationin both ER-negative with mutant p53 and ER -positive with wild-type p53 [21,22]. Also, NFkB and Akt areimplicated as targets for statins in other studies, in a study conducted by Ghosh-Choudhury and colleagues to find how statins (simvastatin) cause breast cancer cell growth inhibition, their results showed that simvastatin cause inhibition of $N F \kappa B$ with subsequent inhibition of expression and transcription of BclXL which is a strong antiapoptotic protein with derepression of PTEN which is antiproliferative tumor suppressor, also, they found that statins (simvastatin) cause significant inhibition of Akt phosphorylation [23]. Another study implicated NFKB, in which the researcher found that simvastatin produce apoptosis in breast cancer cell lines through RhoA-dependent retention of $\mathrm{NF} \kappa \mathrm{B}$ to the cytosol with subsequent inhibition of BCL-2 which is an anti-apoptotic protein accompanying reduction of AKT1 production and then decrease in PKB/AKT proteinlevel [24], the end results of all these mechanisms and pathways is induction of apoptosis.

\section{Tumor Invasion and Metastasis}

The effect of statins on tumor invasion and metastasis was investigated in several studies and there are evidences support the role of statins in impairment of metastatic processes of the tumor by inhibiting several growth factors and invasion factors responsible for tumor invasion and widespread metastasis (e. g., E-selectin, matrix metalloproteinase and epithelial growth factor) in various of tumor cell lines [5]. In certain breast cancer cell lines, the researcher found that combination of statins (atorvastatin) with bisphosphonate (zoledronic acid) was associated with decrease in bone metastasis through inhibition DKK-1 expressed tumors, these results was supported by results of previous studies which showed that atorvastatin and zoledronic cause inhibition of the Wnt inhibitor DKK-1 in breast cancer cell lines(MDA-MB-231, MCF-7 and T47D) and inhibition of WNT3A induced OPG which is potent inhibitor of osteoclast activity production and inhibition of osteoblast differentiation [25,26]. Also, statin (cerivastatin) was suggested to inhibit invasion and metastasis in the aggressive subtypes through prevention of FPP and GPP synthesis which responsible for Ras and Rho translocation in to the cell membrane with subsequent prevention of tumor proliferation and cellular migration as well as cellular signal transduction associated with cellular motility, also, the study showed that cerivastatin deactivated NFKB related to RhoA inhibition processes, leading to reduction in matrix metalloproteinase and urokinas responsible for cellular invasion and migration, this also was noted using another type of statins (lovastatin) that was investigated to test its effect on the F3II sarcomatoid type of breast carcinoma, a highly aggressive, invasive and metastatic tumor type. It was noted 


\section{Cancer Therapy \& Oncology International Journal}

that itprevented tumor cell attachment and migration in vitro and significantly prolonged the time for tumors appearance and decreased tumor growth and metastasis to the lungs from breast carcinomas in vivo $[27,28]$.

\section{Future Directions}

As stated above, the role of statins as anticancer agents was established in several preclinical studies through its antiproliferative, apoptosis induction, anti-angiogenesis and other properties preventing tumor growth and spread. However, the incorporation of statins in cancer treatment protocols still limited, there are some studies that try to find the effect of statins in treatment of breast cancer by incorporation of statins either alone or in combination with other agent to improve its response and decrease risk of tumor recurrence. Combination of endocrine therapy and cholesterol-lowering medication (CLM) was tested in a recent large observational study by the Breast International Group (BIG) 1-98, in which systemic levels of total cholesterol and use of (CLM) were measured at study entry and every 6 months up to 5.5 years, the authors concluded that cholesterol-lowering medication usage during adjuvant endocrine treatment may have a role in prevention of breast cancer recurrence in hormone receptor-positive early-stage breast cancer and they recommend that these observational results should be addressed in future prospective trials [29]. After postmastectomy radiotherapy of high grade breast cancer tumor (e. g., inflammatory and triple negative types) the local failure was expected, Lacerda et al in their study showed that simvastatin radiosensitizes mammosphere-initiating cells of inflammatory breast cancer(IBC) cell lines (MDA-IBC3, SUM149, SUM190) and of the metaplastic, non-IBC triple-negative receptor cell line (SUM159), they concluded that patients with IBC and triple-negative non-IBC breast cancer have the high rates of local failure, without available known radiosensitizers and they reported significant improvement in local control after post mastectomy radiotherapy among statin users with IBC and significant radio sensitization among triple-negative and IBC cell lines of multiple subtypes using simvastatin, so, they recommend that simvastatin should be justified as a radio sensitizing agent by future prospective clinical trials [30]. Moonindranath et al. [6] in their review reported that triple-negative breast cancer and patients with metastatic bone from breast cancer are associated with high levels of DKK-1 which is a negative prognostic marker. So, they suggest that statins may prove to be a more effective alternative than bisphosphonates when used alone or in combination in prevention of bone resorption resulted from bone metastases in patients with breast cancer [6].

\section{Conclusion}

Statins are commonly prescribed drugs relatively inexpensive and well-tolerated in majority of patients. In the era of anti-cancer research specially breast cancer, there are evidences suggest that statins could be used in treatment and prevention through its anti-proliferative, anti-angiogenic, and anti-metastatic properties. However, there are some debates regarding its optimal dose, safety, side effects when used with chemotherapeutic agents and biomarkers determine its effects. However, the role of statins in breast cancer research shouldn't be neglected in the future studies and the next randomized controlled trials should be focused on the role of statin in improvement of response if combined with commonly used anticancer therapy as chemo, hormonal and radiotherapy.

\section{Reference}

1. Collins R, Armitage J, Parish S, Sleigh P, Peto R, et al. ( 2003) MRC/ BHF Heart Protection Studyof cholesterol-lowering with simvastatin in 5963 people with diabetes: arandomized placebo-controlled trial. Lancet 361: 2005-2016.

2. Durazzo AE, Machado FS, Ikeoka DT, De Bernoche C, Monachini MC, et al. (2004) Reduction in cardiovascularevents after vascular surgery with atorvastatin: a randomized trial. J Vasc Surg 39(5): 967-975.

3. Bellosta S, Ferri N, Bernini F, Paoletti R, Corsini A (2000) Non-lipidrelated effects of statins. Ann Med 32(3):164-176.

4. Garwood ER, Kumar AS, Baehner FL, Moore DH, Au A, et al. (2010) Fluvastatin Reduces Proliferation and Increases Apoptosis in Women with High Grade Breast Cancer. Breast Cancer Research and Treatment 119: 137- 144 .

5. Hindler K, Cleeland CS, Rivera E, Collard CD (2006) The Role of Statins in Cancer Therapy .The Oncologist 11(3): 306-315.

6. Moonindranath S, Shen HL (2016) Statins and Breast Cancer: An Overview of the Current Situation. Advances in Breast Cancer Research 5: 14-29.

7. Niemi M, Pasanen MK, Neuvonen PJ (2011) Organic Anion Transporting Polypeptide 1B1: A Genetically Polymorphic Transporter of Major Importance for Hepatic Drug Uptake. Pharmacological Reviews 63: 157-181.

8. Ieiri I, Suwannakul S, Maeda K, Uchimaru H, Hashimoto K, et al. (2007) SLC01B1 (OATP1B1, an Uptake Transporter) and ABCG2 (BCRP, an Efflux Transporter) Variant Alleles and Pharmacokinetics of Pitavastatin in Healthy Volunteers. Clinical Pharmacology and Therapeutics 82(5): 541-547.

9. Hamelin BA, Turgeon J (1998) Hydrophilicity/Lipophilicity: Relevance for the Pharmacology and Clinical Effects of HMG-CoA Reductase Inhibitors. Trends in Pharmacological Sciences 19: 26-37.

10. Wejde J, Hjertman M, Carlberg M, Egestad B, Griffiths WJ, et al (1998) Dolichol-like lipids with stimulatoryneffect on DNA synthesis: substrates for protein dolichylation? J CellBiochem71(4): 502-514.

11. Soma MR, Corsini A, Paoletti R (1992) Cholesterol and mevalonic acid modulationin cell metabolism and multiplication. Toxicol Lett 64-65 Spec No:1-15.

12. Fritz G, Brachetti C, Bahlmann F, Schmidt M, KainaB. (2002) Rho GTPases in Human Breast Tumours: Expression and Mutation Analyses and Correlation with Clinical Parameters. British Journal of Cancer 87(6): 635-644.

13. Keyomarsi K, Sandoval L, Band V, Pardee AB (1991) Synchronization of Tumor and Normal Cells from G1 to Multiple Cell Cycles by Lovastatin. Cancer Research 51(13): 3602-3609.

14. Kanugula AK, Gollavilli PN, Vasamsetti SB, Karnewar S, Gopoju R, et al. (2014) Statin-Induced Inhibition of Breast Cancer Proliferation and Invasion Involves Attenuation of Iron Transport: Intermediacyof Nitric Oxide and Antioxidant Defence Mechanisms. The FEBS Journal 281(16): 3719-3738. 


\section{Cancer Therapy \& Oncology International Journal}

15. Bjarnadottir O, Romero Q, Bendahl PO, Jirstrom K, Ryden Let, al (2013) Targeting HMG-CoA Reductase with Statins in a Windowof-Opportunity Breast Cancer Trial. Breast Cancer Research and Treatment 138(2): 499-508.

16. Feldt M, Bjarnadottir O, Kimbung S, Jirstrom K, Bendahl PO, et al. (2015) Statin-Induced Anti-Proliferative Effects via Cyclin D1 and p27 in a Window-of-Opportunity Breast Cancer Trial. Journal of Translational Medicine 13: 133

17. Weis M, Heeschen C, Glassford AJ, Cooke JP (2002) Statins have biphasic effects on angiogenesis. Circulation 105(6): 739-745.

18. Frick M, Dulak J, Cisowski J, Józkowicz A, Zwick R, et al. (2003) Statins differentially regulate vascularendothelial growth factor synthesis in endothelial and vascular smoothmuscle cells. Atherosclerosis 170(2): 229-236.

19. Kureishi Y, Luo Z, Shiojima I, Bialik A, Fulton D, et al. (2000) The HMGCoA reductase inhibitor simvastatin activates the protein kinase Akt and promotes angiogenesis in normocholesterolemic animals. Nat Med 6(9): 1004-1010.

20. Koyuturk M, Ersoz M, Altiok N (2007) Simvastatin Induces Apoptosis in Human Breast Cancer Cells: p53 and Estrogen Receptor Independent Pathway Requiring Signalling through JNK. Cancer Letters 250(2): 220-228.

21. Brozovic A, Fritz G, Christmann M, Zisowsky J, Jaehde U, et al. (2004) Long-Term Activation of SAPK/JNK, p38 Kinase and Fas-L Expression by Cisplatin Is Attenuated in Human Carcinoma Cells That Acquired Drug Resistance. International Journal of Cancer 112(6): 974-985.

22. Brantley-Finley C, Lyle CS, Du L, Goodwin ME, Hall T, et al. (2003) The JNK, ERK and p53 Pathways Play Distinct Roles in Apoptosis Mediated by the Antitumor Agents Vinblastine, Doxorubicin, and Etoposide. Biochemical Pharmacology 66(3): 459-469.
23. Gopalan A, Yu W, Sanders BG, Kline K (2013) Simvastatin Inhibition of Mevalonate Pathway Induces Apoptosis in Human Breast Cancer Cells via Activation of JNK/CHOP/DR5 Signaling Pathway. Cancer Letters 329: 9-16.

24. Ghosh-Choudhury N, Mandal CC, Ghosh Choudhury G (2010) Simvastatin InducesDerepression of PTEN Expression via NFkB to Inhibit Breast Cancer Cell Growth. Cellular Signalling 22(5): 749-758.

25. Gobel A, Browne AJ, Thiele S, Rauner M, Hofbauer LC, et al. (2015) Potentiated Suppression ofDickkopf-1 in Breast Cancer by Combined Administration of the Mevalonate Pathway Inhibitors Zoledronic Acid andStatins. Breast Cancer Research and Treatment 154(3): 623-631.

26. Rachner TD, Gobel A, Thiele S, Rauner M, Benad-Mehner P, et al. (2014) Dickkopf-1 Is Regulated by the Mevalonate Cancer. Breast Cancer Research16(1): R20.

27. Alonso DF, Farina HG, Skilton G, Gabri MR, De Lorenzo MS, et al. (1998) Reduction of Mouse Mammary Tumor Formation and Metastasis by Lovastatin, an Inhibitor of the Mevalonate Pathway of Cholesterol Synthesis. Breast Cancer Research and Treatment 50: 83-93.

28. Aberg M, Wickstrom M, Siegbahn A (2008) Simvastatin Induces Apoptosis in Human Breast Cancer Cells in a NFrB-Dependent Manner and Abolishes the Anti-Apoptotic Signaling of TF/FVIIa and TF/FVIIa/ FXa. Thrombosis Research 122(2): 191-202.

29. Borgquist S, Giobbie-Hurder A, Ahern TP, Garber JE, Colleoni M, et al. (2017) Cholesterol, cholesterol-lowering medication use, and breast cancer outcome in the BIG 1-98 study. J ClinOncol.

30. Lacerda L, Reddy JP, Liu D, Larson R, Li L, et al. (2014) Simvastatin radiosensitizes differentiated and stem-like breast cancer cell lines and is associated with improved local control in inflammatory breast cancer patients treated with postmastectomy radiation. Stem Cells Transl Med 3(7): 849-856.

\section{Your next submission with Juniper Publishers will reach you the below assets}

Commons Attribution 4.0 License

DOI: $10.19080 /$ CTOIJ.2017.03.555624 